\title{
Limit cycles bifurcating from a zero-Hopf equilibrium of a 3-dimensional continuous differential system
}

\author{
Sara Kassa · Jaume Llibre · Amar \\ Makhlouf
}

Received: date / Accepted: date

Abstract A zero-Hopf equilibrium of a differential sy
librium point whose linear part has eigenvalues 0 and
provide necessary and sufficient conditions for the existe
cycles bifurcating from a zero-Hopf equilibrium of the foll
Lypschizian differential systems
\[ \begin{array}{l}\dot{x}=y, \\ \dot{y}=z, \\ \dot{z}=-a|x|-y+3 y^{2}-x z-b,\end{array} \]

when $a=b=0$. Note that due to the existence of an absolute value the vector field associated to this system is only Lypschitz. We shall prove that these limit cycles persist for $a b>0$ with $a$ and $b$ sufficiently small, i.e. when the differential system has no equilibria. Additionally we provide an estimation of the size of the bifurcating small limit cycles and also characterize their kind of stability or instability.

We remark that there are no works which study the zero-Hopf bifurcation of nonsmooth differential systems as we do in this paper, and that the algorithm that we use here can be applied for studying the zero-Hopf bifurcation of an arbitrary nonsmooth differential system.

Keywords continuous differential system · periodic orbit · limit cycle · averaging theory $\cdot$ zero-Hopf bifurcation $\cdot$ zero-Hopf equilibrium 2010 Mathematics Subject Classification. 37G15.

S. Kassa and A. Makhlouf

Department of Mathematics, University of Annaba, Laboratory LMA, P.O.Box 12, Annaba 23000, Algeria

E-mail: kassasara03@outlook.com, makhloufamar@yahoo.fr

J. Llibre

Departament de Matematiques, Universitat Autònoma de Barcelona, 08193 Bellaterra,

Barcelona, Catalonia, Spain

E-mail: jllibre@mat.uab.cat 


\section{Introduction and statements of the main results}

Jafari, Sprott and Golpayegani in [7] introduced continuous differential systems without equilibria, and studied the existence of chaotic motions in the dynamics of such systems. Azar et al. [1] motivated by the paper of Jafari, Sprott and Golpayegani consider the continuous differential systems

$$
\begin{aligned}
& \dot{x}=y, \\
& \dot{y}=z, \\
& \dot{z}=-a|x|-y+3 y^{2}-x z-b,
\end{aligned}
$$

with $a>0$ and $b>0$. Since the equation $|x|=-b / a$ has no solution if $a b>0$, differential systems (1) has no equilibrium points when $a b>0$. Azar et al. analyzed the dynamical properties and the synchronization of the differential system (1), and they implemented a physical realization of this differential system in order to illustrate its feasibility.

Our objective is to enrich the dynamical properties of the differential system (1) found in [1], showing that this system can exhibit for convenient values of its parameters a zero-Hopf equilibrium from which can bifurcate two or one limit cycles.

A zero-Hopf equilibrium of system (1) is an equilibrium point whose linear part has eigenvalues 0 and $\pm \omega i$ with $\omega>0$. A limit cycle of system (1) is a periodic orbit isolated in the set of all periodic orbits of system (1).

Define the functions

$$
f_{1}(r, w)=\frac{1}{2 \pi}\left(2 w \sqrt{1-\frac{w^{2}}{r^{2}}}-\pi r w+2 r \arcsin \left(\frac{w}{r}\right)\right),
$$

and

$$
f_{2}(r, w)=-\frac{1}{\pi}\left(\pi \beta+2 r \sqrt{1-\frac{w^{2}}{r^{2}}}-2 \pi r^{2}+2 w \arcsin \left(\frac{w}{r}\right)\right) .
$$

Then our main result is the following one.

Theorem 1 Consider the Lipchitz differential system (1) with $a=\varepsilon, b=\varepsilon^{2} \beta$ and $|\varepsilon|$ sufficiently small. Then for each zero $\left(r_{0}, w_{0}\right)$ with $r_{0}>0$ of the function $\left(f_{1}, f_{2}\right)$ such that the determinant of the Jacobian matrix $\partial\left(f_{1}, f_{2}\right) / \partial(r, w)$ evaluated at $\left(r_{0}, w_{0}\right)$ is non-zero, there exist a limit cycle $(x(t, \varepsilon), y(t, \varepsilon), z(t, \varepsilon))$ of system (1) such that

$$
(x(0, \varepsilon), y(0, \varepsilon), z(0, \varepsilon))=\varepsilon\left(r_{0}+w_{0}, 0,-r_{0}\right)+O\left(\varepsilon^{2}\right) .
$$

If the two eigenvalues of the matrix

$$
\left.\frac{\partial\left(f_{1}, f_{2}\right)}{\partial(r, w)}\right|_{(r, w)=\left(r_{0}, w_{0}\right)}
$$

have negative real parts then the periodic solution $(x(t, \varepsilon), y(t, \varepsilon), z(t, \varepsilon))$ is stable. If some of the eigenvalues has positive real part the periodic solution $(x(t, \varepsilon), y(t, \varepsilon), z(t, \varepsilon))$ is unstable. 
Theorem 1 is proved in section 3 using the averaging theory for computing periodic solutions developed in [2] and [3] for Lipschitz differential systems. We remark that the classical averaging theory for computing periodic solutions needs that the differential system be of class $C^{2}$, see for instance Theorems 11.5 and 11.6 of the book of Verhulst [9]. $[5]$.

For another study of the limit cycles of a Lipschitz system see for instance

Corollary 1 Consider the Lipchitz differential system (1) with $a=\varepsilon, b=\varepsilon^{2} \beta$ and $|\varepsilon|$ sufficiently small.

(a) System (1) has two periodic solutions $\left(x_{ \pm}(t, \varepsilon), y_{ \pm}(t, \varepsilon), z_{ \pm}(t, \varepsilon)\right)$ such that

$$
\begin{aligned}
& \left(x \pm(0, \varepsilon), y_{ \pm}(0, \varepsilon), z_{ \pm}(0, \varepsilon)\right)=\varepsilon\left(r_{ \pm}, 0,-r_{ \pm}\right)+O\left(\varepsilon^{2}\right), r_{ \pm}=\frac{1 \pm \sqrt{1+2 \pi^{2} \beta}}{2 \pi}, \\
& \text { if } 1+2 \pi^{2} \beta>0,1-\sqrt{1+2 \pi^{2} \beta}>0 \text { and } 1+2 \pi^{2} \beta-7 \sqrt{1+2 \pi^{2} \beta} \neq 0 .
\end{aligned}
$$

(b) System (1) has one periodic solutions $(x(t, \varepsilon), y(t, \varepsilon), z(t, \varepsilon))$ such that

$$
(x(0, \varepsilon), y(0, \varepsilon), z(0, \varepsilon))=\varepsilon\left(r_{+}, 0,-r_{+}\right)+O\left(\varepsilon^{2}\right), r_{+}=\frac{1+\sqrt{1+2 \pi^{2} \beta}}{2 \pi},
$$

if $1+2 \pi^{2} \beta>0,1-\sqrt{1+2 \pi^{2} \beta}<0$ and $1+2 \pi^{2} \beta-7 \sqrt{1+2 \pi^{2} \beta} \neq 0$.

(c) If additional to the assumptions of statements (a) and (b) we have that $-1-2 \pi^{2} \beta+7 \sqrt{1+2 \pi^{2} \beta}>0$, then the periodic solution associated to the zero $\left(r_{+}, 0\right)$ of the function $\left(f_{1}, f_{2}\right)$ is unstable.

Corollary 1 is also proved in section 3 using Theorem 2 .

\section{The averaging theory for Lipschitz differential systems}

In this section we present the basic results on the averaging theory for the Lipschitz differential systems that we need for proving our main result.

The averaging theory reduces the problem of finding isolated periodic solutions to the problem of finding zeros of a function, called the averaged function. We need the averaging theory of first order for differential systems developed in [2] and [3] whose associated vector field is Lipschiz but not $C^{1}$. The sufficient conditions for the existence of simple isolated zeros of the averaged function are given in terms of the Brouwer degree, see [6] for precise definitions.

The classical averaging theory for finding isolated periodic solutions of a differential system needs that the vector field associated to the system be $C^{2}$. See for general results on averaging theory the book of Sanders, Verhulst and Murdock [8].

Theorem 2 We consider the following differential system

$$
\dot{\mathbf{x}}(t)=\varepsilon F(t, \mathbf{x})+\varepsilon^{2} R(t, \mathbf{x}, \varepsilon),
$$


where $F: \mathbb{R} \times D \rightarrow \mathbb{R}^{n}, R: \mathbb{R} \times D \times\left(-\varepsilon_{f}, \varepsilon_{f}\right) \rightarrow \mathbb{R}^{n}$ are continuous functions, $T$-periodic in $t$, and $D$ is an open subset of $\mathbb{R}^{n}$. We define $f: D \rightarrow \mathbb{R}^{n}$ as

$$
f(\mathbf{z})=\frac{1}{T} \int_{0}^{T} F(s, \mathbf{z}) d s,
$$

and assume that

(i) $F$ and $R$ are locally Lipschitz in $\mathbf{x}$;

(ii) for $\mathbf{a} \in D$ with $f(\mathbf{a})=0$, there exists a neighborhood $V$ of $\mathbf{a}$ such that $f(\mathbf{z}) \neq 0$ for all $\mathbf{z} \in \bar{V} \backslash\{\mathbf{a}\}$ and $d_{B}(f, V, \mathbf{a}) \neq 0$ (where $d_{B}(f, V, \mathbf{a})$ denotes the Brouwer degree of $f$ in the neighborhood $V$ of $\mathbf{a})$.

Then for $|\varepsilon|>0$ sufficiently small, there exists an isolated $T$-periodic solution $\mathbf{x}(t, \varepsilon)$ of system (3) such that $\mathbf{x}(0, \varepsilon) \rightarrow \mathbf{a}$ as $\varepsilon \rightarrow 0$.

If the averaged function $f(\mathbf{z})$ is $C^{1}$ in some neighborhood of a fixed isolated zero a of $f(\mathbf{z})$, then we can use the following remark in order to verify the hypothesis $(i i)$ of Theorem 2 . For more details see again [6].

Remark 1 Let $f: D \rightarrow \mathbb{R}^{n}$ be a $C^{1}$ function, with $f(\mathbf{a})=0$, where $D$ is an open subset of $\mathbb{R}^{n}$ and $\mathbf{a} \in D$. If $\mathbf{a}$ is a simple zero of $f$, i.e the determinant $(\operatorname{det}(D f(\mathbf{a})) \neq 0)$ of the Jacobian matrix of the function $f$ at $\mathbf{a}$ is not zero, then there exists a neighborhood $V$ of a such that $f(\mathbf{z}) \neq 0$ for all $\mathbf{z} \in \bar{V} \backslash\{\mathbf{a}\}$. Then $d_{B}(f, \mathbf{a}, V, 0) \in\{-1,1\}$.

In [3] Theorem 2 is improved as follows.

Theorem 3 Under the assumptions of Theorem 2, for small $\varepsilon$ the condition $\operatorname{det}(D f(\mathbf{a})) \neq 0$ ensures the existence and uniqueness of a $T$-periodic solution $\mathbf{x}(t, \varepsilon)$ of system (3) such that $\mathbf{x}(0, \varepsilon) \rightarrow \mathbf{a}$ as $\varepsilon \rightarrow 0$, and if all eigenvalues of the matrix Dh(a) have negative real parts, then the periodic solution $\mathbf{x}(t, \varepsilon)$ is stable. If some of the eigenvalue has positive real part the periodic solution $\mathbf{x}(t, \varepsilon)$ is unstable.

The averaging theory for studying periodic solutions is very useful see for instance [4].

\section{Proofs of the results}

Proof (Proof of Theorem 1) Consider $a=\varepsilon, b=\varepsilon^{2} \beta$ and $\varepsilon \geq 0$ in system (1). We shall write the differential system (1) in the normal form for applying the averaging theory, i.e. in the form (3). Firstly we rescale the variables $(x, y, z)=$ $(\varepsilon X, \varepsilon Y, \varepsilon Z)$, we get

$$
\begin{aligned}
\dot{X} & =Y \\
\dot{Y} & =Z \\
\dot{Z} & =-Y+\varepsilon\left(3 Y^{2}-X Z-|X|-\beta\right)
\end{aligned}
$$


$(X, 0,0)$ are the singular points of the differential system (5) when $\varepsilon=0$. The linear part of system (5) with $\varepsilon=0$ at the origin is written as

$$
\left[\begin{array}{c}
\dot{X} \\
\dot{Y} \\
\dot{Z}
\end{array}\right]=\left[\begin{array}{ccc}
0 & 1 & 0 \\
0 & 0 & 1 \\
0 & -1 & 0
\end{array}\right]\left[\begin{array}{l}
X \\
Y \\
Z
\end{array}\right]
$$

Then we do the change of variables

$$
\left[\begin{array}{l}
u \\
v \\
w
\end{array}\right]=\left[\begin{array}{ccc}
0 & 0 & -1 \\
0 & -1 & 0 \\
1 & 0 & 1
\end{array}\right]\left[\begin{array}{l}
X \\
Y \\
Z
\end{array}\right],
$$

which writes the linear part of system (5) with $\varepsilon=0$ in its real Jordan normal form. We get the next differential system

$$
\begin{aligned}
\dot{u} & =-v+\varepsilon\left(-3 v^{2}-u(u+w)+|u+w|+\beta\right), \\
\dot{v} & =u, \\
\dot{w} & =\varepsilon\left(3 v^{2}+u(u+w)-|u+w|-\beta\right) .
\end{aligned}
$$

Now we pass system (6) to the cylindrical coordinates $(u, v, w)=(r \cos \theta, r \sin \theta, w)$, and we obtain the differential system

$$
\begin{aligned}
\dot{r} & =\varepsilon \cos \theta\left(r^{2} \cos 2 \theta-r w \cos \theta+|w+r \cos \theta|-2 r^{2}+\beta\right), \\
\dot{\theta} & =1-\frac{\varepsilon \sin \theta}{r}\left(r^{2} \cos 2 \theta-r w \cos \theta+|w+r \cos \theta|-2 r^{2}+\beta\right), \\
\dot{w} & =\varepsilon\left(3 r^{2} \sin ^{2} \theta+r \cos \theta(w+r \cos \theta)-|w+r \cos \theta|-\beta\right) .
\end{aligned}
$$

Taking $\theta$ as a new independent variable in the differential system (7), it becomes

$$
\begin{aligned}
\frac{d r}{d \theta} & =\varepsilon \cos \theta\left(r^{2} \cos 2 \theta-r w \cos \theta+|w+r \cos \theta|-2 r^{2}+\beta\right)+O\left(\varepsilon^{2}\right), \\
\frac{d w}{d \theta} & =\varepsilon\left(r^{2}(2-\cos 2 \theta)-|w+r \cos \theta|+r w \cos \theta-\beta\right)+O\left(\varepsilon^{2}\right) .
\end{aligned}
$$

Now we calculate the averaged function using the formula (4) with the notations

$$
\begin{aligned}
\mathbf{x}=\mathbf{z} & =(r, w), \\
t & =\theta, \\
F(t, \mathbf{x}) & =F(\theta, r, w) \\
T & =2 \pi,
\end{aligned}
$$

where $F(\theta, r, w)=\left(F_{1}(\theta, r, w), F_{2}(\theta, r, w)\right)$ is given by

$$
\left(\begin{array}{c}
F_{1}(\theta, r, w) \\
F_{2}(\theta, r, w)
\end{array}\right)=\left(\begin{array}{c}
\cos \theta\left(r^{2} \cos 2 \theta-r w \cos \theta+|w+r \cos \theta|-2 r^{2}+\beta\right) \\
r^{2}(2-\cos 2 \theta)-|w+r \cos \theta|+r w \cos \theta-\beta
\end{array}\right) .
$$


Then the averaged function $f(r, w)=\left(f_{11}(r, w), f_{12}(r, w)\right)$ is

$$
\begin{aligned}
f_{1}(r, w)= & \frac{1}{2 \pi}\left(\int_{0}^{\arccos \left(\frac{-w}{r}\right)} F_{1}(\theta, r, w) d \theta+\int_{\arccos \left(\frac{-w}{r}\right)}^{2 \pi-\arccos \frac{-w}{r}} F_{1}(\theta, r, w) d \theta\right. \\
& \left.+\int_{2 \pi-\arccos \left(\frac{-w}{r}\right)}^{2 \pi} F_{1}(\theta, r, w) d \theta\right), \\
f_{2}(r, w)= & \frac{1}{2 \pi}\left(\int_{0}^{\arccos \left(\frac{-w}{r}\right)} F_{2}(\theta, r, w) d \theta+\int_{\arccos \left(\frac{-w}{r}\right)}^{2 \pi-\arccos \frac{-w}{r}} F_{2}(\theta, r, w) d \theta\right. \\
& \left.+\int_{2 \pi-\arccos \left(\frac{-w}{r}\right)}^{2 \pi} F_{2}(\theta, r, w) d \theta\right),
\end{aligned}
$$

and we obtain

$$
\begin{aligned}
& f_{1}(r, w)=-\frac{r w}{2}+\frac{w \sqrt{1-\frac{w^{2}}{r^{2}}}}{\pi}+\frac{r \arcsin \left(\frac{w}{r}\right)}{\pi}, \\
& f_{2}(r, w)=-\frac{1}{\pi}\left(\pi \beta-2 \pi r^{2}+2 r \sqrt{1-\frac{w^{2}}{r^{2}}}+2 w \arcsin \left(\frac{w}{r}\right)\right) .
\end{aligned}
$$

Then going back through the change of variables from Theorem 2 and 3 it follows Theorem 1 . We solve the system $\left(f_{1}(r, w), f_{2}(r, w)\right)=(0,0)$ with respect to the variables $r$ and $w$ which is equivalent to solve the system

$$
\begin{gathered}
2 w \sqrt{1-\frac{w^{2}}{r^{2}}}-\pi r w+2 r \arcsin \left(\frac{w}{r}\right)=0, \\
\pi \beta+2 r \sqrt{1-\frac{w^{2}}{r^{2}}}-2 \pi r^{2}+2 w \arcsin \left(\frac{w}{r}\right)=0,
\end{gathered}
$$

By Theorem 2 each zero $\left(r_{0}, w_{0}\right)$ of the averaged function $\left(f_{1}(r, w), f_{2}(r, w)\right)$ given in (9) whose determinant

$$
\left.\operatorname{det}\left(\frac{\partial\left(f_{1}(r, w), f_{2}(r, w)\right)}{\partial(r, w)}\right)\right|_{\left(r_{i}, w_{i}\right)} \neq 0
$$

provides a limit cycle $(r(\theta, \varepsilon), w(\theta, \varepsilon))$ such that

$$
(r(0, \varepsilon), w(0, \varepsilon))=\left(r_{0}, w_{0}\right)+O(\varepsilon),
$$

of system (8), or equivalently a limit cycle $(r(t, \varepsilon), \theta(t, \varepsilon), w(t, \varepsilon))$ such that

$$
(r(0, \varepsilon), \theta(0, \varepsilon), w(0, \varepsilon))=\left(r_{0}, 0, w_{0}\right)+O(\varepsilon),
$$

of the differential system (7), and consequently a limit cycle $(u(t, \varepsilon), v(t, \varepsilon), w(t, \varepsilon))$ such that

$$
(u(0, \varepsilon), v(0, \varepsilon), w(0, \varepsilon))=\left(r_{0}, 0, w_{0}\right)+O(\varepsilon),
$$

of system (6). So we have a limit cycle $(X(t, \varepsilon), Y(t, \varepsilon), Z(t, \varepsilon))$ such that

$$
(X(0, \varepsilon), Y(0, \varepsilon), Z(0, \varepsilon))=\left(r_{0}+w_{0}, 0,-r_{0}\right)+O(\varepsilon),
$$


of system (5). Finally we obtain a limit cycle $(x(t, \varepsilon), y(t, \varepsilon), z(t, \varepsilon))$ such that

$$
(x(0, \varepsilon), y(0, \varepsilon), z(0, \varepsilon))=\varepsilon\left(r_{0}+w_{0}, 0,-r_{0}\right)+O(\varepsilon),
$$

of system (1). This limit cycle tend to the singular point localized at the origin of coordinates when $\varepsilon \rightarrow 0$.

Now the rest of the proof of the theorem follows directly from Theorem 3.

Proof (Proof of Corollary 1) The averaged function $f_{1}\left((r, w), f_{2}(r, w)\right)$ given in (9) has the two zeros $\left(r_{ \pm}, 0\right)$ with $r_{ \pm}=\frac{1 \pm \sqrt{1+2 \pi^{2} \beta}}{2 \pi}$. The determinant (10) at these two zeros takes the values

$$
d_{ \pm}=\frac{1+2 \pi^{2} \beta \mp 7 \sqrt{1+2 \pi^{2} \beta}}{2 \pi^{2}} .
$$

So by Theorem (1) statements (a) and (b) of Corollary 1 follow. Since the eigenvalues of the matrix $(2)$ evaluated at $\left(r_{+}, 0\right)$ are

$$
\pm \frac{-1-2 \pi^{2} \beta+7 \sqrt{1+2 \pi^{2} \beta}}{2 \pi^{2}}
$$

by Theorem (1) it follows that the periodic solution defined by $\left(r_{+}, 0\right)$ is unstable. On the other hand since the eigenvalues of the matrix (2) evaluated at $\left(r_{-}, 0\right)$ are purely imaginary we cannot say anything about its stability or instability.

Acknowledgements The author is partially supported by the Ministerio de Ciencia, Innovación y Universidades, Agencia Estatal de Investigación grants MTM2016-77278-P (FEDER) and PID2019-104658GB-I00 (FEDER), the Agència de Gestió d'Ajuts Universitaris i de Recerca grant 2017SGR1617, and the H2020 European Research Council grant MSCA-RISE2017-777911.

\section{Conflict of interest}

The authors declare that they have no conflict of interest.

\section{References}

1. A.T. Azar, C. Volos, N.A. Gerodimos, G.S. Tombras, V.-T. Pham, A.G. Radwan, S. Vaidyanathan, A. Ouannas and J.M. Munoz-Pacheco, A novel chaotic system without equilibrium: Dynamics, synchronization and circuit realization, Hindawi Complexity, Article ID 7871467, 11 pages (2017)

2. A. Buică and J. Llibre, Averaging methods for finding periodic orbits via Brouwer degree, Bull. Sci. Math. 128, 7-22 (2004)

3. A. Buică, J. Llibre and O.Yu. Makarenkov, On Yu.A.Mitropol'skii's Theorem on periodic solutions of systems of nonlinear differential equations with nondifferentiable right-hand sides Doklady Math. 78, 525-527 (2008) 
4. T. Carvalho, R.D. Euzébio, J. Llibre and D.J. Tonon, Detecting periodic orbits in some $3 \mathrm{D}$ chaotic quadratic polynomial differential systems, Discrete and Continuous Dynamical Systems-Series B 21, 1-11 (2016)

5. J. Llibre and A. Makhlouf, Zero-Hopf bifurcationin the generalized Michelson system, Chaos, Solitons and Fractals, 89, 228-231 (2016)

6. N.G. Lloyd, Degree Theory, Cambridge University Press, (1978)

7. S. Jafari, J.C. Sprott and S.M.R.H. Golpayegani, Elementary quadratic chaotic flows with no equilibria, Physics Letters A 377, 699-702 (2013)

8. J.A. Sanders, F. Verhulst and J. Murdock, Averaging methods in nonlinear dynamical systems, Second edition, Applied Mathematical Sciences 59, Springer, New York, (2007)

9. F. Verhulst, Nonlinear differential equations and dynamical systems, Universitex, Berlin, Springer-Verlag, (1990) 